\title{
Photoconductance of organic single-molecule contacts
}

\author{
J. K. Viljas, ${ }^{1,2, *}$ F. Pauly, ${ }^{1,2}$ and J. C. Cuevas ${ }^{3,1,2}$ \\ ${ }^{1}$ Institut für Theoretische Festkörperphysik and DFG-Center for Functional Nanostructures, \\ Universität Karlsruhe, D-76128 Karlsruhe, Germany \\ ${ }^{2}$ Forschungszentrum Karlsruhe, Institut für Nanotechnologie, D-76021 Karlsruhe, Germany \\ ${ }^{3}$ Departamento de Física Teórica de la Materia Condensada, \\ Universidad Autónoma de Madrid, E-28049 Madrid, Spain
}

(Dated: February 1, 2008)

\begin{abstract}
We study the dc conductance of organic single-molecule contacts in the presence of external electromagnetic radiation (photoconductance). In agreement with previous predictions, we find that the radiation can lead to large enhancements of the conductance of such contacts by bringing off-resonant levels into resonance through photoassisted processes. In our analysis we make use of the simplifying fact that, under certain assumptions, the photoconductance can be expressed in terms of the transmission function in the absence of the radiation. The conductance enhancement is demonstrated for oligophenylene molecules between gold electrodes, whose electronic structure is calculated based on density-functional theory. It is shown that the exponential decay of the conductance with the length of the molecule can be replaced by a length-independent value in the presence of radiation.
\end{abstract}

PACS numbers: 73.63.-b, 73.50.Pz, 73.63.Rt, 73.40.Jn

The use of electromagnetic radiation, such as laser light, can provide a convenient "handle" for controlling the conductance of atomic and single-molecule contacts $\stackrel{1}{\stackrel{1}{*}}$ It has, for example, been demonstrated that by applying light of a certain frequency, some photochromic molecules can be made to change their conformation even when contacted to metallic electrodes $\stackrel{2,3}{2}$ Devices based on such molecules could act as molecular optoelectronic switches ${ }^{4,5,6.7}$ According to theoretical predictions, it may also be possible to construct molecular electronic devices based on other radiation-induced phenomena,,$\underline{8,9,10,11,12,13}$ and several groups are currently working towards experiments of this type $\underline{14,15,16}$

One of the first things to be addressed in interpreting conductance measurements on atomic-sized contacts exposed to external radiation is the role played by heating effects, such as electronic excitations and thermal expansion, 14.17 Another phenomenon to be considered is the excitation of local plasmonic modes and related field-enhancement effects $\stackrel{17,18}{\underline{10}}$ Due to the complexity of the problem, no comprehensive theory exists at the moment. However, we have recently put forward a description based on photoassisted transport $\underline{19}$ We found that, depending on the metal and the radiation frequency, the effect of irradiation on atomic contacts can be either an increase or a decrease in the conductance. We also demonstrated that the approximate behavior of the photoconductance can be predicted based on the transmission function $T(E)$ in the absence of the radiation, without resorting to complicated numerical simulations of the ac-driven transport.

In this brief report we discuss the possible outcome of experiments with laser-irradiated organic single-molecule contacts between two metallic electrodes. We shall argue that for junctions where the Fermi energy of the metal lies in the gap between the highest occupied (HOMO) and lowest unoccupied (LUMO) molecular orbital, photoassisted processes can lead to enhancements of the dc conductance by orders of magnitude. This conclusion has already been made previously, $\underline{\underline{8}}$ but here we want to describe the origin of the effect based on the properties of $T(E)$, along the lines of Ref. 19. We demonstrate the effect for oligophenylene contacts with varying numbers of phenyl rings, whose electronic structure is calculated using density-functional theory. While in the absence of radiation the conductance decays exponentially, in the presence of radiation it may become almost independent of the length of the molecule. This effect should become important already for light frequencies lower than those needed for internal transitions between the HOMO and LUMO levels. $\stackrel{20}{.0}$

In a typical experiment with laser-irradiated atomicsized contacts, the laser spot diameters are on the order of micrometers $\stackrel{14}{ }$ It is therefore much more likely that an incoming photon interacts with the metallic electrode than with the molecule itself. However, at frequencies below the plasma frequency and the electronic interband transition threshold, most of the incoming light is reflected. This is the result of a collective screening response of the electrons. If the light is polarized along the axis of the contact, this generates an oscillating voltage over the contact at the frequency $\omega$ of the radiation. The photoassisted transport through the contact can then be described in terms of "sidebands", where the Fermi-level electrons appear to approach the contact at energies shifted from the equilibrium Fermi level by an integer multiple of the photon energy $\hbar \omega$ (see Refs. 8, 19, 21, 22, 23, 24, 25, 26). In this view, the role of the molecule is, most importantly, to provide the transmission landscape according to which the incoming electrons will be transmitted or reflected. Thus the photoassisted transport can be seen as probing the transmission func- 
tion at energies away from the Fermi level.

Assuming a symmetric junction, low temperature, a double-step voltage profile (i.e. vanishing ac electric field on the molecule), as well as "wide-band" leads, the zerobias dc conductance in the presence of external radiation of frequency $\omega$ (i.e. the photoconductance) can be described by the expression 19

$$
G_{d c}(\omega)=G_{0} \sum_{l=-\infty}^{\infty}\left[J_{l}(\alpha / 2)\right]^{2} T\left(E_{F}+l \hbar \omega\right)
$$

Here $G_{0}=2 e^{2} / h$ is the quantum of conductance, $E_{F}$ is the Fermi energy, $l$ is the sideband index, and $J_{l}(x)$ are Bessel functions of the first kind. Their argument involves $\alpha=e V_{a c} / \hbar \omega$, where $V_{a c}$ is the amplitude of the induced ac voltage. The latter is unknown in practice, because it depends on the environment of the junction, the polarization of the light,, 27 and the frequency itself. Especially if $\omega$ happens to be in resonance with local plasmonic excitations, $V_{a c}$ can be strongly enhanced. For our purposes it is sufficient to treat $\alpha$ simply as a parameter. In the metallic atomic contacts described in Ref. 19 the transmission functions are rather flat, and so the changes in conductance typically remain on the order of a few percent for reasonable ac amplitudes. In contrast, molecular junctions often exhibit large gaps in $T(E)$, corresponding roughly to the energy region between the HOMO and LUMO orbitals of the isolated molecule. The Fermi energy lies somewhere in the gap and thus the dc conductance of the junction in the absence of radiation is very low. It is then to be expected from Eq. (11) that an external frequency corresponding to the smaller of the energy differences between $E_{F}$ and the two gap edges can lead to a considerable enhancement of the conductance. This is because the photoassisted processes essentially change the character of the transport from off-resonant to resonant tunneling. $\underline{.}$

The photoassisted conductance-enhancement described above should be contrasted with the lightinduced (rectification) currents studied in Ref. 20, for example. The latter are due to a direct internal pumping of electrons between the HOMO and LUMO levels of a molecule coupled weakly and asymmetrically to the electrodes. Such internal transitions may become dominant for frequencies exceeding the HOMO-LUMO gap. In contrast, the photoassisted processes described by Eq. (11) involve photon emission and absorption in the contact regions between the molecule and the electrodes, while the transport on the molecule is assumed to be elastic. These processes should set in already for $\hbar \omega$ smaller than half of the gap. In the following we consider low enough frequencies, such that the internal electronic transitions can be assumed to be unimportant. On the other hand, in order to apply Eq. (1), we must consider (approximately) symmetric contacts. Therefore, significant rectification effects will be absent in any case.

To illustrate the above ideas for realistic molecules, we have used density-functional theory (DFT) to describe (a)
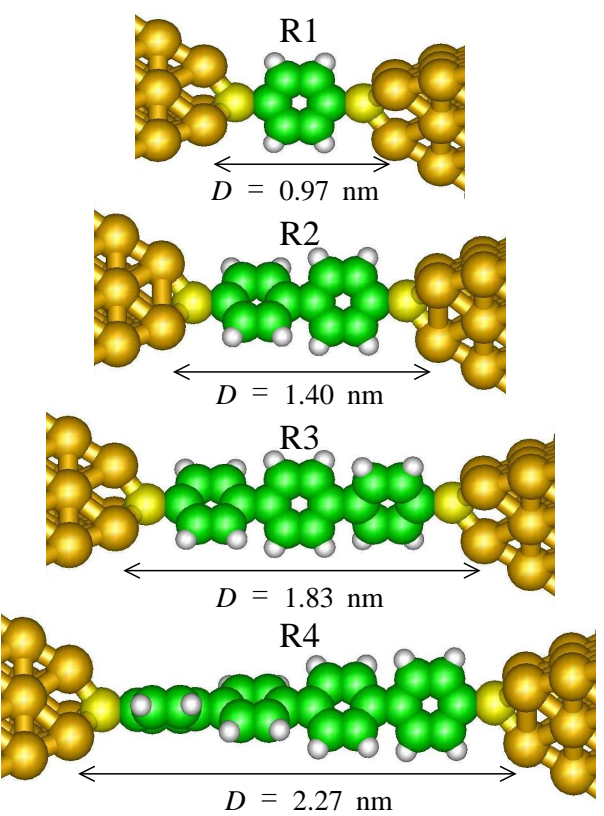

(b)

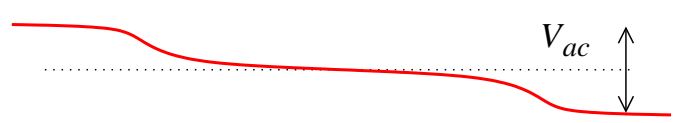

FIG. 1: (Color online) (a) The four molecular contacts R1R4 we have studied, containing oligophenylenes with one to four phenyl rings and coupled to $\mathrm{Au}$ [111] pyramids through sulfur atoms. (b) Our model assumes the induced ac voltage $V_{a c}$ to drop in a double-step manner, as illustrated here schematically for contact R4.

oligophenylene molecules with varying numbers of phenyl rings bridging two gold electrodes. The description of the electronic structure and the relaxation of the geometries is done using the TURBOmOLE quantum chemistry package, $\stackrel{28}{2}$ and the transmission functions are calculated using Green's function techniques 29,30 The four molecular junctions considered here are shown in Fig. 1(a). They are formed of oligophenylenes containing one to four phenyl rings and are bonded to the fcc [111] gold pyramids through sulfur atoms. We refer to them as $\mathrm{R} 1$ to $\mathrm{R} 4$, according to the number of rings. The corresponding isolated molecules have HOMO-LUMO gaps of $3 \mathrm{eV}$ or more, and thus to induce internal transitions of electrons between the HOMO and LUMO levels would require energies corresponding to blue or ultraviolet light. On the other hand, the photoassisted effects we are describing are expected to take effect already in the red or infrared part of the spectrum. Note that the double-step ac voltage profile assumed by Eq. (10) is not unreasonable, since one can expect the voltage to drop abruptly at the sulfur atoms due to partial screening of the electric field on the molecule $13,31,32,33,34,35$ See Fig. 1(b) for an illustration.

Figure 2(a) shows the $T(E)$ functions for the four molecular contacts R1 to R4. All of them feature a gap on the order of the HOMO-LUMO gap of the iso- 

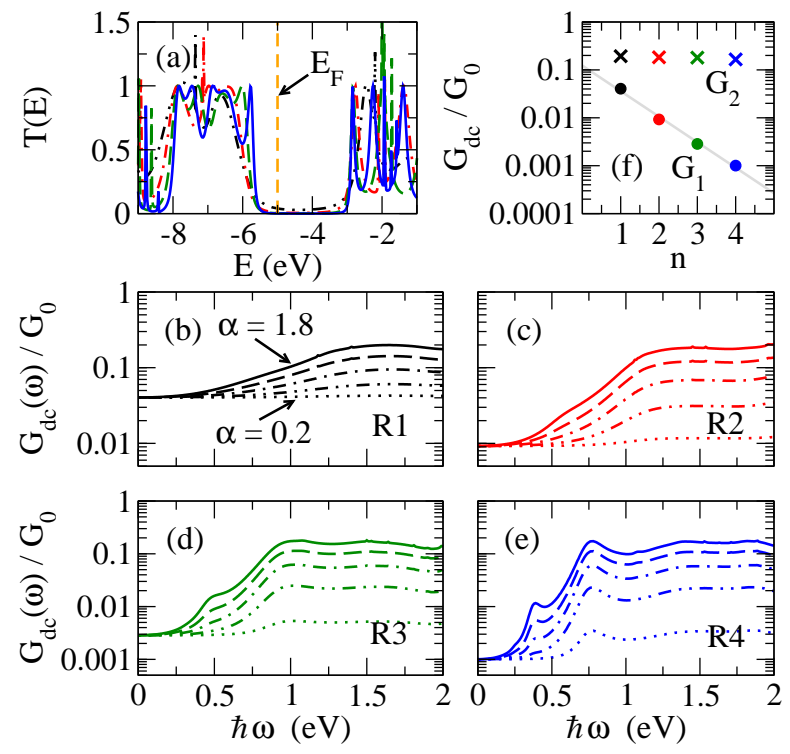

FIG. 2: (Color online) (a) Transmission versus energy $[T(E)]$ for the contacts R1-R4 in Fig. 1 (dash-dot-dotted, dashdotted, dashed, and solid lines, respectively). (b)-(e) The photoconductance versus external frequency $\omega$ for the contacts R1-R4, respectively. For each case the results for the following values of $\alpha$ are shown: $0.2,0.6,1.0 ., 1.4$, and 1.8, in order of increasing conductance. (f) The dc conductances in the absence $\left(G_{1}\right.$, dots) and presence $\left(G_{2}\right.$, crosses) of radiation with $\hbar \omega=1.5 \mathrm{eV}$ and $\alpha=1.8$ for an increasing number $n$ of phenyl rings. The gray line is a fit of the $G_{1}$ results to an exponential law (see text).

lated molecules. The Fermi energy is in the gap, somewhat closer to the HOMO than the LUMO edge. The panels (b)-(e) present the full results for $G_{d c}(\omega)$ for several representative values of $\alpha$, as obtained from Eq. (1). Note that the zero-frequency results reproduce the conductances in the absence of radiation, i.e. $G_{d c}(\omega=0)=$ $G_{0} T\left(E_{F}\right)$ independently of $\alpha$. When $\hbar \omega$ is increased to values above the energy difference between $E_{F}$ and the HOMO edge of the gap, $G_{d c}(\omega)$ can increase by orders of magnitude as one of the sidebands comes into resonance with the molecular energy levels $[l=-1$ in Eq. (1)]. For $\alpha \gtrsim 1$ also "two-photon" processes $(l=-2)$ begin to contribute, such that the conductance enhancement begins already at lower frequencies (see Ref. 36). In Fig. 2(f) we present the results for $G_{d c}(\omega)$ in the absence of radiation $\left(G_{1}\right.$, dots) and in the presence of radiation with $\hbar \omega=1.5 \mathrm{eV}$ and $\alpha=1.8\left(G_{2}\right.$, crosses $)$ for an increasing number $n$ of phenyl rings. The value of $G_{1}$ exhibits an exponential decay, characteristic of off-resonant tunneling. The gray line represents a fit to $G_{1} \propto G_{0} \exp (-\beta D)$, where $D$ is the distance between the gold tips (see Fig. 1). We obtain an attenuation factor of $\beta=2.8 \mathrm{~nm}^{-1}$, in agreement with previous experimental and theoretical results $\frac{37,38}{}$ In contrast, $G_{2}$ is almost independent of $n$, because the photoassisted processes change the character of the transport to resonant tunneling. Thus the conduc- tance enhancement due to radiation is bigger for larger $n$.

We have also performed calculations with oligophenylene contacts, where the conjugation of the molecules has been broken by side groups. $T(E)$ then exhibits sharp resonances, leading to large fluctuations in $G_{d c}(\omega)$. The maximal conductance enhancements can, however, be even much larger than those in Fig. (2).

It must be emphasized that the calculations of the photoconductance are based on ground-state DFT and Eq. (1), which introduce several simplifying assumptions. First, a proper treatment of the electronic structure in the presence of time-dependent fields should be based on more advanced techniques $\frac{13,39}{3}$ Second, the ac-driven transport cannot in general be described in terms of independent sidebands,$\underline{8,22,23,24,40}$ Nevertheless, detailed tight-binding calculations $\frac{19}{}$ for atomic contacts reproduce the essential features of results obtained with Eq. (11). In particular, the shape of the assumed ac voltage profile [see Fig. 1(b)] does not seem to be crucial. As an additional check, we have studied chain models describing contacts with gaps in $T(E)$ (not shown). In these simulations, a linear ramp-like ac voltage profile (a constant ac electric field) tends to reduce the conductance enhancement, but it still remains an order-of-magnitude effect. Note that such a potential profile was also assumed in Ref. 8 . Indeed, in the case of molecular junctions, the light-induced increase in the dc conductance can be so huge that it is difficult to see how it could be completely washed away in a more rigorous treatment. The best chances of measuring the effect in experiments would be at infrared frequencies, such that the absorption of light and associated heating effects are minimal.

Finally, it is worth noting that the photoconductance $G_{d c}(\omega)$ should not be confused with an ac conductance. The latter quantity has also been discussed recently in the context of molecular contacts. $\stackrel{13.41}{ }$ It is more difficult to describe theoretically as well as to measure reliably, since the capacitance of the junction and hence displacement currents will play a more important role than in the case of the dc response.

In conclusion, we discussed the effect of external radiation on the transport properties of organic singlemolecule contacts between metallic electrodes, where the Fermi energy lies in a gap of the transmission function. The importance of the collective response of the leads to the radiation was emphasized, as compared with the internal electronic transitions due to a direct pumping of the molecule. We have discussed how, under certain assumptions, the photoconductance can be related to the transmission function of the contact in the absence of the radiation. This relation was used to analyze the radiation-induced conductance enhancement for oligophenylene molecules of varying lengths in gold contacts. It was shown that the exponential decay of the dc conductance can be replaced by a length-independent behavior as a result of the photoassisted transport processes. 
We acknowledge helpful discussions with Daniel Guhr, Elke Scheer, Paul Leiderer, Marcelo Goffman, and Jan van Ruitenbeek. The Quantum Chemistry group of Reinhart Ahlrichs is thanked for providing us with TuR-
BOMOLE. This work was financially supported by the Helmholtz Gemeinschaft (Contract No. VH-NG-029), by the DFG within the CFN, and by the EU network BIMORE (Grant No. MRTN-CT-2006-035859).
* Electronic address: janne.viljas@kit.edu

1 T.-H. Lee, J. J. Gonzalez, J. Zheng, and R. M. Dickson, Acc. Chem. Res. 38, 534 (2005).

2 D. Dúlic, S. J. van der Molen, T. Kudernac, H. T. Jonkman, J. J. D. de Jong, T. N. Bowden, J. van Esch, B. L. Feringa, B. J. van Wees, Phys. Rev. Lett. 91, 207402 (2003); S. J. van der Molen, H. van der Vegte, T. Kudernac, I. Amin, B. L. Feringa, and B. J. van Wees, Nanotechnology 17, 310 (2006); N. Katsonis, T. Kudernac, M. Walko, S. J. van der Molen, B. J. van Wees, B. L. Feringa, Advanced Materials 18, 1397 (2006).

3 J. He, F. Chen, P. A. Liddell, J. Andréasson, S. D. Straight, D. Gust, T. A. Moore, A. L. Moore, J. Li, O. F. Sankey, S. M. Lindsay, Nanotechnology 16, 695 (2005).

4 J. Li, G. Speyer and O. F. Sankey, Phys. Rev. Lett. 93, 248302 (2004).

5 C. Zhang, M.-H. Du, H.-P. Cheng, X.-G. Zhang, A. E. Roitberg and J. L. Krause, Phys. Rev. Lett. 92, 158301 (2004); C. Zhang, Y. He, H.-P. Cheng, Y. Xue, M. A. Ratner, X.-G. Zhang, and P. Krstic, Phys. Rev. B 73, 125445 (2006).

6 M. Zhuang and M. Ernzerhof, Phys. Rev. B 72, 073104 (2005).

7 J. Huang, Q. Li, H. Ren, H. Su, and J. Yang, J. Chem. Phys. 125, 184713 (2006).

8 A. Tikhonov, R. D. Coalson, Y. Dahnovsky, J. Chem. Phys. 116, 10909 (2002); J. Chem. Phys. 117, 567 (2002).

9 J. Lehmann, S. Kohler, P. Hänggi, and A. Nitzan, Phys. Rev. Lett. 88, 228305 (2002); J. Lehmann, S. Camalet, S. Kohler, and P. Hänggi, Chem. Phys. Lett. 368, 282 (2003).

10 I. Urdaneta, A. Keller, O. Atabek, and V. Mujica, Int. J. Quant. Chem. 99, 460 (2004); J. Phys. B: At. Mol. Opt. Phys. 38, 3779 (2005).

11 S. Welack, M. Schreiber, and U. Kleinekathöfer, J. Chem. Phys. 124, 044712 (2006).

12 E. R. Bittner, S. Karabunarliev, and A. Ye, J. Chem. Phys. 122, 034707 (2005).

13 R. Baer, T. Seideman, S. Ilani, and D. Neuhauser, J. Chem. Phys. 120, 3387 (2004).

14 D. Guhr, D. Rettinger, J. Boneberg, A. Erbe, P. Leiderer, and E. Scheer, cond-mat/0612117.

15 C. Meyer, J. M. Elzermann, and L. P. Kouwenhoven, Nano Lett. 7, 295 (2007).

16 J. M. Simmons, I. In, V. E. Campbell, T. J. Mark, F. Léonard, P. Gopalan, and M. A. Eriksson, Phys. Rev. Lett. 98, 086802 (2007).

17 S. Grafström, J. Appl. Phys. 91, 1717 (2002).

18 A. W. Sanders, D. A. Routenberg, B. J. Wiley, Y. Xia, E. R. Dufresne, and M. A. Reed, Nano Lett. 6, 6, 1822 (2006).

19 J. K. Viljas and J. C. Cuevas Phys. Rev. B 75, 075406 (2007).

20 M. Galperin and A. Nitzan, Phys. Rev. Lett. 95, 206802
(2005); J. Chem. Phys. 124, 234709 (2006).

21 P. K. Tien and J. P. Gordon, Phys. Rev. 129, 647 (1963).

22 M. H. Pedersen and M. Büttiker, Phys. Rev. B 58, 12993 (1998).

23 M. Moskalets and M. Büttiker, Phys. Rev. B 69, 205316 (2004).

24 S. Kohler, J. Lehmann, and P. Hänggi, Phys. Rep. 406, 379 (2005).

25 G. Platero and R. Aguado, Phys. Rep. 395, 1 (2004).

26 J. C. Tucker and M. J. Feldman, Rev. Mod. Phys. 57, 1055 (1985).

27 We assume the polarization direction to be along the molecular bridge, since this should give the largest values of $V_{a c}$.

28 R. Ahlrichs, M. Bär, M. Häser, H. Horn, and C. Kölmel, Chem. Phys. Lett. 162, 165 (1989).

29 F. Pauly, Ph.D. thesis, Institut für Theoretische Festkörperphysik, Universität Karlsruhe, Karlsruhe (2007).

30 S. Wohlthat, F. Pauly, J. K. Viljas, J. C. Cuevas, and G. Schön, cond-mat/0702477 F. Pauly et al., to be published (2007).

31 S. Datta, W. Tian, S. Hong, R. Reifenberger, J. I. Henderson, and C. P. Kubiak, Phys. Rev. Lett. 79, 2530 (1997).

32 N. D. Lang and Ph. Avouris, Phys. Rev. Lett. 84, 358 (2000).

33 V. Mujica, A. E. Roitberg, and M. A. Ratner, J. Chem. Phys. 112, 6834 (2000).

34 P. S. Damle, A. W. Ghosh, and S. Datta, Phys. Rev. B 64, 201403(R) (2001).

35 A. Nitzan, M. Galperin, G.-L. Ingold, and H. Grabert, J. Chem. Phys. 117, 10837 (2002).

36 We only show results for small enough $\alpha$ and $\omega$, such that the associated electric fields remain of reasonable magnitude. The maximum values for $V_{a c} / D$ for the results in Fig. 2 are on the order of $10^{9} \mathrm{~V} / \mathrm{m}$. In experiments, typical incident powers per area of the focused laser beam are $10^{7}-10^{8} \mathrm{~W} / \mathrm{m}^{2}$ (Refs. 14 25), which correspond to considerably smaller fields of $10^{5} \mathrm{~V} / \mathrm{m}$. However, due to the fieldenhancement effects mentioned above, the field strengths described by $\alpha$ can exceed the externally applied fields by orders of magnitude ${ }^{17}$

37 D. J. Wold, R. Haag, M. A. Rampi, and C. D. Frisbie, J. Phys. Chem. B 106, 2813 (2002).

38 M. Kondo, T. Tada, K. Yoshizawa, J. Phys. Chem. A 108, 9143 (2004).

39 S. Kurth, G. Stefanucci, C.-O. Almbladh, A. Rubio, and E. K. U. Gross, Phys. Rev. B 72, 035308 (2005).

${ }^{40}$ L. Arrachea and M. Moskalets, Phys. Rev. B 74, 245322 (2006).

41 J. Wu, B. Wang, J. Wang and H. Guo, Phys. Rev. B 72, 195324 (2005). 